\title{
Systematic identification of factors bound to isolated metaphase ESC chromosomes reveals a role for chromatin repressors in compaction
}

Dounia Djeghloul ${ }^{1}$, Anne-Céline Kohler ${ }^{1}$, Bhavik Patel ${ }^{2}$, Holger Kramer ${ }^{3}$, Nicolas Veland ${ }^{1}$, Chad Whilding ${ }^{4}$, Andrew Dimond ${ }^{1}$, James Elliott ${ }^{2}$, Amelie Feytout ${ }^{1}$, Tanmay A.M. Bharat ${ }^{5}$, Abul K. Tarafder ${ }^{5}$, Jan Löwe ${ }^{6}$, Bee L. $\mathrm{Ng}^{7}$, Ya Guo ${ }^{1}$, Karen Brown ${ }^{1}$, Jacky Guy ${ }^{8}$, Matthias Merkenschlager ${ }^{1}$ and Amanda G. Fisher ${ }^{1,9, *}$

${ }^{1}$ Lymphocyte Development Group,

${ }^{2}$ Flow Cytometry Facility,

${ }^{3}$ Biological Mass Spectrometry and Proteomics Facility,

${ }^{4}$ Microscopy Facility,

MRC London Institute of Medical Sciences, Imperial College School of Medicine, Hammersmith Hospital Campus, Du Cane Road, London, W12 0NN, UK

${ }^{5}$ Sir William Dunn School of Pathology, University of Oxford, OX1 3RE, UK

${ }^{6}$ MRC Laboratory of Molecular Biology, Cambridge, UK

${ }^{7}$ Wellcome Sanger Institute, Wellcome Genome Campus, Hinxton, Cambridge, CB10 1SA, UK

${ }^{8}$ The Wellcome Centre for Cell Biology, University of Edinburgh, Edinburgh, EH93BH, UK

${ }^{9}$ Lead contact

*Correspondence: amanda.fisher@Ims.mrc.ac.uk 


\begin{abstract}
Epigenetic information is transmitted from mother to daughter cells through mitosis. To identify trans-acting factors and cis-acting elements that might be important for conveying epigenetic memory through cell division, we isolated native (unfixed) chromosomes from metaphasearrested cells using flow cytometry and performed LC-MS/MS to determine the repertoire of chromosome-bound proteins. Quantitative proteomic comparisons between metaphasearrested cell lysates and chromosome-sorted samples revealed a cohort of proteins that were significantly enriched on mitotic ESC chromosomes. These include pluripotency-associated transcription factors, repressive chromatin-modifiers (such as PRC2 and DNA methyltransferases) and proteins governing chromosome architecture. We showed that deletion of PRC2, DNMT1/3a/3b or Mecp2 provoked an increase in the size of individual mitotic chromosomes consistent with de-condensation, as did experimental cleavage of cohesin complexes. These data provide a comprehensive inventory of chromosome-bound factors in pluripotent stem cells at mitosis and reveal an unexpected role for chromatin repressor complexes in preserving mitotic chromosome compaction.
\end{abstract}




\section{Introduction}

Cell division requires genetic and epigenetic information to be accurately duplicated and conveyed to daughter cells. This process relies upon DNA synthesis at S-phase and the subsequent segregation of copies during mitosis $(\mathrm{M})$ phase. In recent years, huge progress has been made in understanding not only how chromosomal DNA is copied and segregated, but also how epigenetic information is transmitted through the cell cycle. For example, we know that DNA methylation is reinstated during S-phase through the activity of DNMT1, an enzyme that re-establishes methylation at hemi-methylated $\mathrm{CpG}$ sites $^{1,2}$. It has also been proposed that during S-phase the epigenetic modifier Polycomb Repressor Complex 2 (PRC2) can both methylate histone $\mathrm{H} 3$ at lysine 27 (through Ezh2) and recognise this mark (through Eed) ${ }^{3}$, ensuring that histone H3K27 methylation is copied at newly synthesised DNA strands ${ }^{4}$. Understanding how epigenetic information is then transmitted through mitosis, as newly replicated genomes condense and segregate, is the subject of intense investigation but remains only partially understood. Progressive activation of CyclinB1-Cdk1 promotes chromosome condensation ${ }^{5}$ so that visibly discrete individual mitotic chromosomes appear at the mitotic spindle ahead of breakdown of the nuclear envelope. The physical basis of this chromosome compaction, as cells move from interphase into metaphase, remains somewhat controversial with evidence supporting at least two different models. The hierarchical model describes an ordered folding of DNA into consecutive higher-order structures, beginning with the $11 \mathrm{~nm}$ chromatin fibre representing simple nucleosome arrays (so-called beads on a string $)^{6,7}$. A second model, originally proposed by Paulson and Laemmli, ${ }^{8}$ and supported by recent findings ${ }^{10}$, evokes a chromosome scaffold that comprises a continuous core of nonhistone proteins running along the axis of the chromosome, from which radial loops of chromatin are attached. Although discrete in their architectures, both models envisage the formation of mitotic chromosomes as a multi-layered process requiring specialised proteins (such as condensin 1 and 2) and bespoke modifications to histone tails (such as histone H3S10 phosphorylation) that collectively drive spatial compression via close-range and more distant interactions ${ }^{11}$. While these interactions differ from those at interphase, it seems likely that at least some factors mediating local chromatin condensation during interphase might have similar or related roles in mitosis. The cohesin complex, for example, is required to keep newly replicated sister chromatids in close physical proximity during S/G2 to M phases of the cell cycle, but can also modulate interphase gene expression through local enhancer-promoter contact $^{12-17}$.

Transmission of gene expression features from mother to daughter cells is also associated with DNA sequence-specific transcription factor binding through cell division ${ }^{18}$. Although it was originally thought that most sequence-specific transcription factors were likely to be displaced 
from chromosomes at mitosis ${ }^{19}$, subsequent studies focused on the Hsp70 gene promoter ${ }^{20}$, or that have examined the dynamic distribution of GATA1, FOXAI and ESRRB in cycling cells ${ }^{21-23}$ have shown that these factors remain bound to mitotic chromosomes and occupy a subset of the genomic sites bound in interphase ${ }^{23,24}$. In this setting, the continued binding of such factors has been proposed to 'bookmark' the mitotic genome, marking out specific genes for subsequent activity in daughter cells. Over the last few years, the number of mitotic bookmarking factors described has increased and now includes transcription factors associated with pluripotency (such as Oct4, Sox2 and KIf4) as well as more ubiquitously expressed chromatin-modifiers (such as Brd4, MII, Ring1a and Bmi1) ${ }^{23,25-31}$. Studies have also shown that the retention of DNA binding factors on mitotic chromosomes can occur through interactions with a variety of emergent features of condensed mitotic chromatin ${ }^{32-35}$, rather than exclusively through cognate DNA motifs. To comprehensively evaluate the repertoire and roles of mitotic chromosome-binding proteins in dividing pluripotent ESCs we wanted to move away from candidate-based analysis towards higher-throughput and unbiased approaches. Technical difficulties associated with successfully synchronising cells, ensuring that mitotic samples do not contain interphase contaminants, and a requirement to independently validate 'chromosome-bound' components from among heterogeneous mitotic lysates, have limited the performance of many conventional approaches. In addition, an increased appreciation that fixatives intended to stabilize or cross-link mitotic preparations can artificially displace factors from native mitotic chromosomes ${ }^{28,36,37}$, infer that prior studies might have substantially underestimated the repertoire of factors that bind to condensed mitotic chromosomes in vivo. To circumvent these issues, we directly isolated native (unfixed) mitotic chromosomes from dividing ES cells using Hoechst 33258 and Chromomycin A3 staining of DNA and flow cytometry to enumerate and sort specific chromosomes on the basis of their AT/GC content and forward scatter. This builds upon prior approaches ${ }^{38-40}$ enabling mitotic chromosome purification from different cell types and species. By performing LC-MS/MS analysis on conventional metaphase-arrested ESCs (lysates), in parallel with highly-enriched (sorted) chromosomes, we were able to catalogue the factors present in mitotic ESCs and discriminate chromosome-bound factors as being significantly enriched in chromosome-sorted fractions (Figure 1). From among 5,888 proteins reproducibly detected in mitotic ESC lysates, only around $10 \%$ (614) were significantly enriched on purified mitotic chromosomes. These included transcription factors (such as Sox2, Esrrb and Sall4), members of the structural maintenance of chromosomes family of proteins (such as Smc1), heterochromatin-associated proteins and repressive chromatin modifiers such as DNA methyl-transferases, PRC1 and PRC2. Importantly, we showed that individual mitotic chromosomes isolated from ESCs lacking PRC2 activity, DNA methylation or Mecp2, were significantly de-condensed relative to equivalent chromosomes from wild type (WT) ESCs, providing a functional validation for the 
roles of these components on mitotic chromosomes. In addition, metaphase chromosomes isolated from differentiated cells were more compact than equivalents isolated from ESCs and these isolated chromosomes remained responsive to biologically-relevant cues. In situ cleavage of endogenous cohesin complexes provoked de-condensation and a loss of structural integrity. Our study therefore illustrates a new approach in studying the properties of mitotic chromosomes and reveals an inventory of chromosome-bound proteins that comprise the mitotic signature of mouse pluripotent stem cells. 


\section{Results}

\section{Isolation of native metaphase chromosomes from mouse ESCs}

We adapted a protocol used previously to isolate unfixed mitotic chromosomes ${ }^{40}$. Briefly, rapidly dividing cultures of mouse ESCs were arrested in metaphase using demecolcine to achieve samples where most (85-90\%) of cells were in M-phase as judged by PI labelling (Figures 1a and S1a). Condensed chromosomes were released using polyamine buffer, stained with Hoechst 33258 and Chromomycin A3 as described previously ${ }^{40-42}$, and examined by flow cytometry using a Becton Dickinson influx equipped with specialised air-cooled lasers (see detailed methods). This approach allows individual chromosomes to be discerned and either sorted en mass (upper plot, Figure 1a) or gated on individual chromosomes such as the $X$ or chromosome 19 (highlighted separately in lower plot, Figure 1a). After sorting, the integrity of sorted chromosome was examined and verified by optical imaging using antibody to CENPA or using TRF1-YFP to confirm centromere and telomere number and location (Figure S1b).

\section{Analysis of proteins bound to isolated metaphase ESC chromosomes}

To determine the proteins protein bound to native (unfixed) metaphase ESC chromosomes we performed a proteomic analysis using LC-MS/MS and analysed the data using the labelfree quantification (LFQ) algorithm within the MaxQuant software platform. In these experiments we compared samples with equivalent numbers of ESC metaphase chromosomes $\left(10^{7}\right)$ before and after chromosome sorting, in three biological replicates. This led to the identification of 5888 proteins in mitotic lysates of which 5436 were identified with two or more razor or unique peptides per protein at a $1 \%$ false discovery rate (FDR). Our rationale was that chromosome-bound factors should be enriched in sorted samples, while factors that were not chromosome-associated would be depleted. In the sorted fractions we detected 3750 proteins (Figure $1 \mathrm{~b}$ ) that were either significantly enriched as compared to mitotic lysates $(614$, red), were depleted relative to mitotic lysates $(1548$, blue) or showed no statistical difference between the two (1488, grey) (Figures $1 \mathrm{~b}$ and $1 \mathrm{c}$ ). Consistent with our expectations, we found histones significantly enriched in the sorted samples (Figure 1d, Red) whereas components of the proteasome and members of the minichromosome maintenance complex $(\mathrm{Mcm})$ were depleted from mitotic chromosomes (Figures $1 \mathrm{e}$ and $1 \mathrm{f}$ respectively, blue). Mcm components are critical for initiating DNA synthesis but are known to dissociate from chromosomes once DNA synthesis has occurred in S-phase and cells transit into $G 2^{43}$. The relative depletion of $\mathrm{Mcm}$ factors among mitotic chromosome-sorted samples provides further confidence that chromosome-bound and non-bound proteins can be discriminated using this approach. Further analysis of the proteomic data (Figure S1c) showed that 
chromatin remodelling complexes, chromosome architectural proteins and selected DNA binding proteins were enriched in chromosome-sorted samples. Importantly, among the transcription factors that regulate ESC pluripotency and differentiation, enrichment on mitotic chromosomes was selective (Figure 1g). For example, Utf1, a transcription factor required for the proper differentiation of embryonic carcinoma and embryonic stem cells ${ }^{44}$ was enriched in sorted mitotic chromosome samples, while Nanog, Oct4 (Pou5f1) and Klf4, although detected, showed no significant enrichment on sorted chromosomes. Esrrb and Sox2, two previously characterised bookmarking factors ${ }^{23,24,26-28}$ were significantly enriched in chromosome-sorted samples, while factors such as Dppa5 and KIf5 were significantly depleted from chromosomesorted fractions, implying that they might be evicted from chromosomes during mitosis. Antibody-mediated labelling confirmed abundant Sox2 bound to isolated mitotic ESC chromosomes (Figure S1d), while Oct4 and Nanog appeared low or undetectable, consistent with the proteomic analysis. Proteins associated with gene repression and heterochromatin formation including the HMTases Suv39h1 and Suv39h2 and components of PRC1 and PRC2 such as Jarid2, Eed and Ezh2, were all significantly enriched in chromosome-sorted samples (Figure $1 \mathrm{~h}$ and supplemental table1). The DNA methyltransferases Dnmt1, Dnmt3a and Dnmt3b also showed co-enrichment after chromosome sorting, suggesting that most of the chromatin machinery required to sustain $\mathrm{H} 3 \mathrm{~K} 9 \mathrm{me} 3, \mathrm{H} 3 \mathrm{~K} 27 \mathrm{me} 3$ and $5 \mathrm{mC}$ marking of the genome remains bound to ESC chromosomes through mitosis. Likewise, the methyl-CpGbinding protein Mecp2, and the SWI/SNF related protein Smarca5 were also significantly enriched in chromosome-sorted samples (Figure 1h). Smarca5 is an important chromatin remodelling factor that is involved in establishing regularly spaced nucleosomes, critical for DNA replication and repair, and associated with both positive and negative transcriptional outcomes. Smarca5 has been shown to interact with Rad2145, and enrichment of Rad21, Smc1, Smc2 and other SMC-associated proteins was evident among chromosome-sorted samples (Figure 1i, red), consistent with these factors also remaining bound to ESC metaphase chromosomes. Rad21 binding to isolated ESC chromosomes was confirmed by antibody labelling (Figure S1d) showing a centromeric distribution with lower abundance along chromosome arms.

\section{DNA methylation and PRC2 activity keep mitotic chromosomes compact}

To investigate the relevance of these chromatin-based repressors we examined mitotic chromosomes from ESCs that lack DNA methylation ${ }^{46}$ or PRC2 activity ${ }^{47}$. Although the overall distribution of mitotic chromosomes isolated from WT and mutant ESCs (Dnmt1,3a, $3 b^{-/-}$or $E e d^{-1}$ ) was similar (Figure 2a), a close inspection revealed differences in the size and shape of these mitotic chromosomes. To accurately measure this, we separately purified two representative mitotic chromosomes (19 and X) from WT and mutant ESCs, using Hoechst 33258 and Chromomycin A3 staining and flow sorting as described previously (Figure 1a). 
DNA-FISH with mouse chromosome 19- or X-specific paints (Figure S2a) confirmed 99-100\% sample purity. The size of individual chromosomes was estimated by microscopy using standard imaging software to determine the total chromosome area and estimate the size of DAPI-bright pericentric domains (Figure S2b). These analyses indicated that mitotic 19 and $X$ chromosomes isolated from Dnmt1,3a, $3 b^{-/}\left(23.4 \pm 4.1,43.6 \pm 6.9 \mu \mathrm{m}^{2}\right.$ respectively) or Eed ${ }^{-/-}$ $\left(24.4 \pm 4.8, \quad 42.6 \pm 4.7 \mu \mathrm{m}^{2}\right.$ respectively) ESCs were significant larger than equivalent chromosomes isolated from WT ESCs $\left(18.4 \pm 3.1,34.6 \pm 5.3 \mu \mathrm{m}^{2}\right.$ respectively) (Figures $2 \mathrm{~b}$ and 2c). In contrast, mitotic chromosomes isolated from a Sox2-deficent ESC line ${ }^{48}$ were comparable in size and shape to equivalent mitotic chromosomes isolated from WT ESCs (Figure $2 \mathrm{~b}$ and $2 \mathrm{c}$ ). These data suggest that DNA methylation and PRC2 activity are important for efficient chromosome condensation in mitosis. Mitotic chromosomes from WT ESCs showed a distribution of $\mathrm{H} 3 \mathrm{~K} 27 \mathrm{me} 3$ along the entire chromosome, with centromeres decorated by histone H3K9me3 (Figure S2e). Dnmt1,3a, $3 b^{-/-}$mutants lacked $5 \mathrm{mC}$ and had reduced levels of both $\mathrm{H} 3 \mathrm{~K} 9 \mathrm{me} 3$ and H3K27me3 (mid panel Figure S2c-d and middle panel S2e), while Eed mutant lacked H3K27me3 but retained H3K9me3 and 5mC (Figure S2c-d and right panel S2e).To exclude that chromosome de-compaction seen in PRC2-deficient ESCs was not due to inadvertent secondary effects, we examined Eed ${ }^{-1-}$ ESCs transfected with a BAC that contains Eed (clone B1.3BAC) that had previously been shown to restore PRC2 activity and H3K27me ${ }^{47}$. As shown in Figure 2d, metaphase chromosomes isolated from Eed-rescued (Eed-BAC) were similar in size and shape to equivalent chromosomes from WT ESCs, confirming that the de-compaction seen in Eed-null chromosomes was fully reversed by restoring PRC2 activity.

\section{Mecp2 contributes to the mitotic compaction of autosomes in ESCs}

To determine whether the methyl binding protein Mecp2 contributes to mitotic chromosome condensation we examine ESCs lacking Mecp2 ${ }^{49}$. Mecp2 has been implicated in regulating chromatin architecture at a range of different levels, from the juxtaposition of nucleosome arrays, to condensing pericentric heterochromatin, and is reported to interact with a plethora of partners independent of binding methylated $\mathrm{CpG}$ (reviewed $\mathrm{in}^{50}$ ). Comparing metaphase chromosomes 19, 3 and $X$ isolated from parental male Mecp2-expressing (Mecp2 $2^{\text {lox/y }}$ ) and Mecp2-deficient $\left(M e c p 2^{-/ y}\right)$ ESCs we observed a significant increase in the sizes of both autosomes in the absence of Mecp2, but no apparent change in the size of active $X$ chromosomes (Figure $3 a$ and $3 b$ ). Although CpG methylation is found on active and inactive $X$ chromosomes, published ChIPseq analysis indicates that MeCp2 is less abundant on $X$ and $Y$ chromosomes than on autosomes (Figure S3a and S3b) in ESCs and in neurons ${ }^{51,52}$. Depletion of Mecp2 provoked a profound change in the distribution of histone H3K9me3 along metaphase chromosomes. In particular, dense H3K9me3 labelling that normally is confined 
to pericentric (DAPI-intense) regions on metaphase chromosomes, extended along the arms of autosomes in the absence of Mecp2 (Figure 3c, lower panel, quantified in S3c and S3d). This spread in $\mathrm{H} 3 \mathrm{~K} 9 \mathrm{me} 3$ distribution beyond DAPI-intense regions, was not evident in equivalent chromosomes isolated from Dnmt1,3a, $3 b^{-/-}$or Eed ${ }^{-/} \mathrm{ESCs}$, and H3K9me3 was much reduced in Dnmt1,3a, 3b-/ ESCs (Figure S2e, green).

\section{Mitotic chromosome size depends upon cell context and differentiation state}

As PRC2 activity and DNA methylation are required for successful differentiation but not for ESC self-renewal or pluripotency (reviewed $\mathrm{i}^{53,54}$ ), we asked whether mitotic chromosomes isolated from differentiated cells and ESCs were similar. To examine this, we isolated individual chromosomes from metaphase arrested mouse ESCs (Figure 4a), pre-B cells (Figure $4 \mathrm{~b}$ ), mouse cardiomyocyte HL-1 cells (Figure 4c) and primary embryo fibroblasts (Figure $4 \mathrm{~d}$ ). Although the success of metaphase arrest varied between the different cell types (45\%-90\%), by applying a FACS-based approach we were able to isolate and purify mitotic chromosomes, irrespective of differences arising from cell cycle synchronisation or karyotype complexity. Using chromosomes 19 and $X$ as representatives, we found that mitotic chromosomes isolated from differentiated pre-B cells, cardiomyocytes and fibroblasts, were significantly smaller than equivalents isolated from ESCs (Figure $4 \mathrm{e}$ and $4 \mathrm{f}$ ). As these size differences could reflect constraints imposed earlier in the cell cycle, for example by the size of nuclei, we measured the diameter of G1- and G2-phase nuclei in each of the different cell types (Figure S4a). This comparison showed similar nuclear sizes in each of the different cell types examined in G1 (Figure S4b), or in G2-phases (Figure S4c).

\section{Isolated metaphase chromosomes are sensitive to in situ cohesin cleavage}

A major potential advantage of purifying native chromosomes from cells in mitosis is that these unfixed chromosomes may respond to appropriate biological cues when applied 'in situ'. To test this, we asked whether experimental cleavage of cohesin could significantly alter the structure of isolated chromosomes. Cohesin complexes are composed of Smc1, Smc3, the kleisin Scc1, and one of three auxillary subunits ${ }^{13}$ and are required to keep sister chromatids together from DNA replication until mitosis, as well having roles in interphase genome organisation, gene transcription and DNA repair ${ }^{12-17}$. Proteomic analysis (Figure $1 \mathrm{i}$ and S1c) indicate that components of the cohesin complex bind mitotic chromosomes in different cell types $^{25,55,56}$ and are dynamically regulated so that the majority of cohesin dissociates from chromosome arms during prophase. Some cohesin remains bound at centromeres until anaphase when separase cleaves the kleisin subunit ${ }^{13,57,58}$. We used a pre-B cell line that expresses a cleavable form of Rad21 (Rad21-TEV-myc) to investigate the impact of cohesin complex dissolution ${ }^{59,60}$. We first examined the distribution of Rad21 on native mitotic chromosomes isolated from pre-B cells and from ESCs and, as anticipated, detected 
substantial Rad21 binding at centromeric regions (as illustrated for chromosome 19, Figure $5 \mathrm{a}$ and $\mathrm{S} 5 \mathrm{a}$ ). We then performed Hi-C analysis of flow-sorted mitotic chromosomes from pre$B$ cells to confirm that the 3D chromosome contacts present at interphase (Figure S5b, upper panels) were lost from mitotic chromosome samples (Figure S5b, lower panels), consistent with previous reports ${ }^{10,61}$. We then isolated native mitotic chromosomes from WT and Rad21 ${ }^{\text {Tev/Tev }}$ pre-B cell lines (Figure S5c) and examined the impact of cohesin cleavage induced by TEV protease (Figures 5b). Briefly, metaphase-arrested WT or Rad2 $1^{\text {Tev/Tev }}$ pre-B cells were used to sort mitotic chromosomes en mass, or gated for chromosome 19, and these samples were treated with TEV enzyme $(0.06 \mathrm{U} / \mathrm{ml}$ for 4 hours $)$ or with buffer alone and imaged using advanced optical microscopy or cryo-electron tomography (cryo-ET) (Figure 5c shows the experimental design). TEV treatment resulted in efficient and selective cleavage of Rad21Tev, as verified by Myc immunofluorescence labelling (Figure $5 \mathrm{~d}$ ) and Western blotting (Figure S5d). TEV-induced cohesin cleavage also resulted in a significant increase in the size of mitotic chromosome 19, compared with either untreated (-TEV) or TEV-treated chromosomes derived from WT pre-B cells (Figure 5e). Increased mitotic chromosome 19 size was accompanied by a de-condensation at DAPI-bright pericentric domains (arrowed). Further cryo-ET analysis of two independent experiments (Figure 5f) confirmed that TEV-induced cohesin cleavage resulted an increase in the size of mitotic (Rad21 ${ }^{\text {Tev/Tev }}$ ) chromosome 19 , and a widespread de-condensation was evident in representative 3D images (supplemental videos 1 and 2). 


\section{Discussion}

The isolation and purification of mitotic chromosomes by flow cytometry offers a new approach for studying chromosome structure and dissecting the interplay between transcription factors and chromatin that convey cellular memory through mitosis. We show that the size of individual metaphase chromosomes differs between different cell types, something that we believe has not been reported previously. Native mitotic chromosomes purified from ESCs were much less condensed than equivalents isolated from either lymphocytes, cardiomyocytes or fibroblasts. The idea that chromosomes of pluripotent ESCs might be more 'loosely-packed' than somatic equivalents is consistent with previous studies in interphase in which electron spectroscopic imaging (ESI) revealed that ESCs and cells of the mouse earlyepiblast (E3.5) lack the compact chromatin domains that characterise differentiated lymphocytes, liver and kidney cells ${ }^{62}$. ESI studies have also shown that at later stages of development (E5.5) epiblast cells loose the dispersed 10nm chromatin fibres that are so-called architectural hallmarks of pluripotency ${ }^{63}$. Our ability to purify and compare mitotic chromosomes from stem and differentiating cells will be important for future studies aimed at understanding the basis of these dynamic changes in chromosome structure, and mapping changes in mitotic bookmarking at key developmental transitions. We have also shown that native metaphase chromosomes purified by FACS, remain responsive to appropriate biological cues. As an example, we show that in situ cleavage of residual cohesin that is bound to centromeric domains on metaphase chromosomes, provokes de-condensation and the loss of structural integrity. This infers that metaphase chromosomes isolated in their native state remain sensitive to regulators of chromosome architecture, a finding that should enable the mechanisms of chromosome condensation and de-condensation to be more closely observed. We examined the repertoire of proteins bound to metaphase chromosomes in pluripotent ESCs, using FACS and a quantitative LC-MS/MS approach to discriminate proteins that were significantly enriched on sorted chromosomes, versus 'unbound' proteins in mitotic lysates. This revealed a repertoire of $>600$ proteins that remain bound to metaphase ESC chromosomes, many of which have established roles in chromatin organisation, DNA and nucleosome packaging, cell cycle and chromosome architecture and function. Three subsets of transcription factors relevant for pluripotency were discerned in mitotic ESCs that showed either a significant enrichment on metaphase chromosomes versus lysates (eg. Sox2, Utrf1, Dppa4, Dppa2, Sal4, Esrrb), were depleted (eg. Dppa5, Klf5) or were similarly represented in both (eg. Oct4, Nanog, Klf4). The first group includes Esrrb and Sox2, transcription factors that were previously been shown to remain bound throughout mitosis in ESCs and implicated in mitotic bookmarking ${ }^{23,27,28}$. The later two subgroups included candidates evicted from condensing metaphase chromosomes or that were in dynamic flux so that they appeared to be equivalently distributed between mitotic lysate and chromosomes samples. Examples of 
this include Oct4, Nanog and Klf4 that were detected but showed no significant enrichment after chromosome sorting. Studies by others have shown that Oct4, Nanog and Klf4 can remain bound to metaphase ESC chromosomes ${ }^{26}$, albeit at low levels (Figure S1d). Conceivably differences in these results could reflect subtle changes in transcription factor concentration and DNA binding (on rates) at isolated mitotic chromosomes that may impact occupancy ${ }^{64}$.

Proteomic analysis also showed that many of the core components of Polycomb Repressor Complex 1 (Rnf2, Pcgf6, Cbx2, Phc1) and Polycomb Repressor Complex 2 (Eed, Ezh2, Suz12) that are responsible for catalysing histone H2AK119 mono-ubiquitination and histone K3K27 tri-methylation respectively, were enriched on metaphase ESC chromosomes. Likewise, DNA methyl-transferases (Dnmt1, Dnmt3a and Dnmt3b) and the methyl-CpGbinding protein Mecp2 were also significantly enriched on metaphase chromosomes as compared to lysates. Previous studies had suggested that Polycomb group proteins $\mathrm{PC}, \mathrm{PH}$, PSC and BMI1 dissociate from condensing chromosomes from prophase to metaphase in Drosophila embryos and in human primary cells ${ }^{65,66}$. Studies of living, rather than fixed samples, indicated that although GFP-tagged PC fusion proteins are depleted from mitotic chromosomes relative to interphase, some PC complexes remained bound ${ }^{67}$. Our results were obtained using unfixed but highly purified metaphase ESC chromosomes and resemble data from a recent study of chromatin-bound changes through the cell cycle in human glioblastoma T98G cells ${ }^{25}$ showing that chromatin repressor complexes remained bound throughout mitosis. Among chromatin activator complexes, including those previously linked to stem cell self-renewal and pluripotency ${ }^{68-70}$, we found a wide range of distributions. Some Trithorax (Trx) family members appeared to be enriched on metaphase chromosomes (Kmt2a, Kmt2b, similar to Chd1, Chd2, Chd4, Chd8) while others appeared more abundant in mitotic lysates (Brd4, Ash2l, Wrd5, Wrd82). Previous studies of Trithorax-family proteins during mitosis have reported that MLL can remain bound to ${ }^{31}$ or dissociate from mitotic Hela chromosomes, being rebound upon exit from mitosis ${ }^{71}$. Studies of chromatin activator complexes in Drosophila have also suggested that trithorax proteins such as Ash1, can remain bound to mitotic chromosomes in the embryonic blastoderm while Polycomb-group proteins such as EZ, $\mathrm{PHO}$ and $\mathrm{PC}$ dissociate ${ }^{72}$. While it is possible that complexity and functional redundancy within the trithorax family members ${ }^{73}$ may explain the often discrepant reports of occupancy during mitosis, our data underscore the importance of both cellular context and native (unfixed) samples in defining the metaphase chromatin repertoire.

We demonstrate that Dnmt1,3a,3b, Mecp2, PRC1 and PRC2 repressors remain bound to metaphase chromosomes in pluripotent ESCs. To assess their functional relevance, we examined chromosomes from ESCs lacking DNA methylation, Mecp2, or PRC2 activity (and associated H3K27me3), as compared to those lacking the transcription factor Sox2. Genome- 
wide loss of DNA methylation or $\mathrm{H} 3 \mathrm{~K} 27 \mathrm{me} 3$ resulted in mitotic chromosomes that were larger and less compact than WT equivalents. At first sight this is surprising since in interphase reduced DNA methylation is reported to affect nuclear organisation, histone modifications and linker histone binding, but does not directly alter chromatin compaction ${ }^{74}$. Furthermore, at the nucleosome level, in vitro studies have indicated that DNA methylation alone does not induce chromatin compaction ${ }^{75,76}$. This suggests that the de-compaction of metaphase chromosomes seen in Dnmt1, 3a, 3b-null ESCs most likely stems from secondary changes that serve to relax the higher order structure of chromatin ${ }^{6}$, perhaps through altered histone $\mathrm{H} 1$ binding or an impaired recruitment of Mecp2. Mecp2 is a multifunctional protein that can bind both methylated and un-methylated DNA, can compete with histone $\mathrm{H} 1$, and has been shown to directly mediate nucleosome oligomerisation and compaction in vitro ${ }^{77}$. Consistent with this, metaphase chromosomes isolated from Mecp2-null ESCs also showed a de-condensation of autosomes relative to WT equivalents. The size of the $\mathrm{X}$ chromosome, that is active in WT male ESCs and relatively depleted of Mecp2 as compared to autosomes, was however unaffected. In post mitotic neurons Mecp2 depletion is reported to provoke an increase, rather than a decrease in chromatin compaction, associated with histone H4K2Ome3 being repositioned to pericentric heterochromatin territories previously occupied by Mecp2 ${ }^{78}$. Our experiments have exclusively examined chromosomes during mitosis, where prior withdrawal of DNA methylation, PRC2-mediated H3K27me3, or Mecp2 each separately induced an increase in chromosome size, consistent these heterochromatin-associated features being important for keeping chromosomes compact. Taken together these experiments not only provide a functional validation for chromatin repressors being bound at metaphase chromosomes in ESCs but also highlight the importance of an interplay between chromatin remodellers and trans-acting factors for conveying mitotic memory. 


\section{Materials and Methods}

\section{Cells}

ESCs used in this study were wild type (WT) E14Tg2a ${ }^{48}$, Sox2-deficient (clone 2O5) ${ }^{48}$, Dnmt1,3a, 3b ${ }^{-1-46}, \mathrm{Eed}^{-1-}$ (clone B1.3), rescued Eed null (Eed BAC) ${ }^{47}$, and floxed Mecp2 ESC clones $\left(M e c p 2^{10 x / y}\right.$ and $\left.M e c p 2^{-1 y}\right)$. These clones were generated by transfecting a floxed Mecp2 line with Cre. Mecp2 $2^{-/ y}$ clone has expressed Cre and deleted the floxed segment, Mecp2 $2^{10 x / y}$ clone has been unaltered. ESCs were cultured on $0.1 \%$ gelatine-coated plates. Cells were grown in KO-DMEM medium supplemented with 15\% FCS, non-essential amino acids, Lglutamine, 2-mercaptoethanol, antibiotics and $1000 \mathrm{U} / \mathrm{ml}$ of leukaemia inhibitory factor. Abelson-transformed pre-B cell lines (WT and Rad21 ${ }^{\text {Tev/Tev }}$ pre-B cells were cultured in IMDM medium supplemented with 10\% FCS, $2 \mathrm{mM}$ L-glutamine, antibiotics, and $50 \mu \mathrm{M} 2-$ mercaptoethanol, and non-essential amino acids. These cell were derived in our lab from transgenic Rad21-Tev-Myc mice ${ }^{60}$. These mice express a modified and functional Rad21 protein containing three Tev cleavage sites within the flexible polypeptide connecting the $\mathrm{N}$ terminal and C-terminal domains. A Myc tag was added together with the Tev sites and allow us to follow the cleavage and the chromosomal localisation of Rad21 by an anti-Myc antibody. Transgenic mice were obtained from Nasmyth laboratory (University of Oxford, Department of Biochemistry). Mouse cardiomyocyte cells (HL-1) were cultured in Claycomb medium (Sigma-Aldrich) supplemented with $10 \%$ of FBS (F2442, Sigma-Aldrich), $10 \mu \mathrm{g} / \mathrm{ml}$ Penicillin and Streptomycin, $2 \mathrm{mM}$ L-glutamine, and $0.1 \mathrm{mM}$ Norepinephrine. Mouse embryonic fibroblasts (MEFs) were cultured in DMEM supplemented with $10 \%$ of FCS, $10 \mu \mathrm{g} / \mathrm{ml}$ Penicillin and Streptomycin, 2mM L-glutamine.

\section{Mitotic arrest and cell cycle analysis}

24h after passaging the cells, demecolcine (D1925, Sigma-Aldrich) was added to the culture media to a final concentration of $0.1 \mu \mathrm{g} / \mathrm{ml}$. ESCs and pre-B cells were incubated with demecolcine for $6 \mathrm{~h}$. Fibroblast and cardiomyocyte cells were incubated for $12 \mathrm{~h}$ at $37^{\circ} \mathrm{C}$. Cells were collected before and after metaphase arrest (after mitotic shake off). $10^{6}$ cells were fixed with ice-cold $70 \%$ ethanol, overnight at $-20^{\circ} \mathrm{C}$. Prior to staining, cells were washed twice with PBS and resuspended in the staining buffer containing $0.05 \mathrm{mg} / \mathrm{ml}$ of Propidium lodide $(\mathrm{PI})$, $1 \mathrm{mg} / \mathrm{ml} \mathrm{RNaseA}$, and $0.05 \%$ of NP40. Samples were incubated $10 \mathrm{~min}$ at RT and $20 \mathrm{~min}$ on ice. PI signal was analyzed in a linear mode using BD LSRII flow cytometer and BD DIVA software.

\section{Chromosome preparation and flow sorting}

Chromosomes were prepared using a polyamine-based method as described previously ${ }^{40,41}$. Mitotic cells were collected by mitotic shake off. The cells were centrifuged at $1200 \mathrm{rpm}$ for $5 \mathrm{~min}$ at room temperature (RT). The cell pellets were gently resuspended in $5-10 \mathrm{ml}$ of hypotonic solution ( $75 \mathrm{mM} \mathrm{KCl}, 10 \mathrm{mM}$ MgSO4, 0.5mM Spermidine, 0.2mM Spermine; $\mathrm{pH}=8$ ) 
for $15 \mathrm{~min}$ at RT. The swollen cells were then centrifuged at $1500 \mathrm{rpm}$ for $5 \mathrm{~min}$ at RT and resuspended in $1-3 \mathrm{ml}$ of freshly prepared ice-cold polyamine isolation buffer $(15 \mathrm{mM}$ Tris- $\mathrm{HCl}$, $2 \mathrm{mM}$ EDTA, $0.5 \mathrm{mM}$ EGTA, $80 \mathrm{mM} \mathrm{KCl}, 3 \mathrm{mM}$ DTT, 0.25\% Triton X-100, $0.2 \mathrm{mM}$ spermine and $0.5 \mathrm{mM}$ spermidine; $\mathrm{pH}=7.5$ ). After $15 \mathrm{~min}$ of incubation on ice, the chromosomes were released by vortexing vigorously for 20-30s. To increase chromosome recovery, the suspension was passed through a 22-gauge needle using a $1 \mathrm{ml}$ syringe. Chromosomes suspension were centrifuged for $2 \mathrm{~min}$ at $1000 \mathrm{rpm}$, RT. Then, the supernatant containing mitotic chromosomes was filtered using a $20-\mu \mathrm{m}$ mesh filter into a 15-ml Falcon tube. Chromosomes were stained at $4^{\circ} \mathrm{C}$ overnight with $5 \mu \mathrm{g} / \mathrm{ml}$ of Hoechst $33258,50 \mu \mathrm{g} / \mathrm{ml}$ of Chromomycin $\mathrm{A} 3$ and $10 \mathrm{mM} \mathrm{MgSO} 4$. At least $1 \mathrm{~h}$ prior chromosome sorting, sodium citrate and sodium sulfite were added to chromosome suspensions to a final concentration of $10 \mathrm{mM}$ and $25 \mathrm{mM}$ respectively. Chromosomes were examined by flow cytometry using a Becton Dickinson Influx equipped with spatially separated air cooled lasers. Hoechst33258 was excited using a (Spectra Physics Vanguard) 355nm laser with a power output of $350 \mathrm{~mW}$. Hoechst33258 fluorescence was collected using a 400nm long pass filter in combination with a $500 \mathrm{~nm}$ short pass filter. Chromomycin A3 was excited using a (Melles Griot) $457 \mathrm{~nm}$ laser with a power output of $300 \mathrm{~mW}$. Chromomycin A3 fluorescence was collected using a $500 \mathrm{~nm}$ long pass filter in combination with a $600 \mathrm{~nm}$ short pass filter. Forward scatter was measured using a (Coherent Sapphire) $488 \mathrm{~nm}$ laser with a power output of $200 \mathrm{~mW}$ and this was used as the trigger signal for data collection. Chromosomes were sorted at an event rate of 15000 per second. A 70 micron nozzle tip was used along with a drop drive frequency set to $\sim 96 \mathrm{KHz}$ and the sheath pressure was set to $65 \mathrm{PSI}$. Isolated chromosomes were collected in DNA lowbinding tubes containing excess of polyamine buffer.

\section{Proteomics}

FACS sorted mitotic chromosomes were digested with trypsin using an in-Stage Tip digestion protocol $^{79}$ and protein digests were analysed by liquid chromatography-tandem mass spectrometry (LC-MS/MS). Data obtained from triplicate experiments were analysed using the Label-Free Quantification algorithm in MaxQuant ${ }^{80}$ and statistical analysis as well as data visualisation were performed using the Perseus software platform ${ }^{81}$.

\section{Immunofluorescence}

Flow-sorted chromosome (chromosomes 19 and X) were spun onto Poly-L-lysine coated slides (VWR) by cytocentrifugation (Cytospin3, Shandon) at 1,300rpm for 10min, RT. Chromosomes samples were blocked with $6 \%$ of normal goat serum for $1 \mathrm{~h}$ at RT and incubated overnight at $4^{\circ} \mathrm{C}$ in a humid chamber with the primary antibodies Cenpa (2040S, Cell Signaling), Rad21 (Ab154769, Abcam), Myc (SC40, Santa Cruz), Sox2 (Ab97959, Abcam), Nanog (REC-RCAB0002P-F, 2bScientific), Oct4 (sc-5279, Santa Cruz). Chromosomes where washed (buffer containing 10mM HEPES, 2mM MgCl2, 100mM KCL, 
and 5mM EGTA) and incubated with appropriate secondary antibodies (anti-mouse-Alexa488 (A11001, Invitrogen), or anti-rabbit-Alexa488 (A11008, Invitrogen), or anti-mouse-A566 (A11031, Invitrogen)) for $1 \mathrm{~h}$ at RT. Immuno-stained chromosomes were mounted in Vectorshield containing DAPI mounting medium. Super-resolution structured illumination (SIM) microscopy was performed on a Zeiss Elyra microscope using a Plan-Apochromat $63 x / 1.4$ oil objective lens.405nm and $488 \mathrm{~nm}$ laser used for fluorescent excitation, and fluorescent emission collected using a bandpass 420-480nm+bandpass, 495-550nm+ longpass $650 \mathrm{~nm}$ filter. Wide-field epi-fluorescence microscopy was performed on an Olympus IX70 inverted microscope using a UPlanApo 100x/1.35 Oil Objective lens.

\section{TEV Protease cleavage}

Total chromosomes $\left(10^{7}\right)$ or chromosomes $19\left(2.10^{5}\right)$ were incubated with or without the AcTEV protease (Invitrogen) (10 units), 4h at RT with gentle rotation, according to manufacturer's instruction. Samples were then collected for western blot and prepared for optical imaging and Cryo-Electron Tomography (Cryo-ET).

\section{Cryo-Electron Tomography}

Samples for cryo-ET were prepared by mixing $10 \mu \mathrm{L}$ of flow sorted Chromosomes 19 with $1 \mu \mathrm{L}$ protein-A conjugated to $10 \mathrm{~nm}$ colloidal gold (CMC Utrecht). $2.5 \mu \mathrm{L}$ of the mixture was pipetted onto freshly glow-discharged Quantifoil Cu/Rh R3.5/1 200 mesh grids and plunge frozen into liquid ethane after removal of excess liquid using a Vitrobot Mark IV (FEI). Frozen grids were transferred to and stored in liquid nitrogen until imaging. Tilt series data was collected on a FEI Titan Krios operating at $300 \mathrm{keV}$, equipped with a Quantum energy filter and a K2 direct electron detector (Gatan) operating in counting mode, using SerialEM software ${ }^{82}$. Tilt series were collected in two directions starting from $0^{\circ}$, at an unbinned calibrated pixel size of $8.4 \AA$ between $\pm 60^{\circ}$ with a $1^{\circ}$ increment at $9 \mu \mathrm{m}$ underfocus. A combined dose of $100 \mathrm{e}^{-} / \AA^{2}$ was applied over the entire series. Tilt series data were aligned and visualised using $\operatorname{IMOD}^{83}$. 


\section{Acknowledgements}

We would like to thank R. Henderson for for experimental suggestions, C. Tyler-Smith for enabling chromosome sorting, T. Adejumo, F. Pereira, P. Chana for expertise and providing reagents, A. Lisini for reading the manuscript and advice. This work was funded by core support from the medical research council UK to the London Institute of Medical Sciences.

\section{Author contributions}

D.D. and A.G.F. conceived and designed the study. D.D. performed most of the experiments, designed the figures and contributed to writing the manuscript. B.P., H.K., N.V., A.D., A.C.K., A.F., C.W., K.B., and G.Y. conducted experiments and performed analysis. T.A.M.B., A.K.T. performed EM imaging. J.L., J.E., J.G., and B.L.N. provided scientific advice and support. M.M contributed to study design and writing manuscript. A.G.F. conceived and designed the study, wrote the manuscript and supervised the experiments. 


\section{References}

1. Hermann, A., Goyal, R. \& Jeltsch, A. The Dnmt1 DNA-(cytosine-C5)-methyltransferase methylates DNA processively with high preference for hemimethylated target sites. $J$ Biol Chem 279, 48350-48359 (2004).

2. Probst, A.V., Dunleavy, E. \& Almouzni, G. Epigenetic inheritance during the cell cycle. Nat Rev Mol Cell Biol 10, 192-206 (2009).

3. Margueron, R. et al. Role of the polycomb protein EED in the propagation of repressive histone marks. Nature 461, 762-767 (2009).

4. Hansen, K.H. et al. A model for transmission of the H3K27me3 epigenetic mark. Nat Cell Biol 10, 1291-1300 (2008).

5. Gavet, O. \& Pines, J. Progressive activation of CyclinB1-Cdk1 coordinates entry to mitosis. Dev Cell 18, 533-543 (2010).

6. Li, G. \& Reinberg, D. Chromatin higher-order structures and gene regulation. Curr Opin Genet Dev 21, 175-186 (2011).

7. Woodcock, C.L. \& Ghosh, R.P. Chromatin higher-order structure and dynamics. Cold Spring Harb Perspect Biol 2, a000596 (2010).

8. Paulson, J.R. \& Laemmli, U.K. The structure of histone-depleted metaphase chromosomes. Cell 12, 817-828 (1977).

9. Maeshima, K. \& Laemmli, U.K. A two-step scaffolding model for mitotic chromosome assembly. Dev Cell 4, 467-480 (2003).

10. Gibcus, J.H. et al. A pathway for mitotic chromosome formation. Science 359 (2018).

11. Antonin, W. \& Neumann, H. Chromosome condensation and decondensation during mitosis. Curr Opin Cell Biol 40, 15-22 (2016).

12. Michaelis, C., Ciosk, R. \& Nasmyth, K. Cohesins: chromosomal proteins that prevent premature separation of sister chromatids. Cell 91, 35-45 (1997).

13. Nasmyth, K. \& Haering, C.H. Cohesin: its roles and mechanisms. Annu Rev Genet 43, 525-558 (2009).

14. van Ruiten, M.S. \& Rowland, B.D. SMC Complexes: Universal DNA Looping Machines with Distinct Regulators. Trends Genet 34, 477-487 (2018).

15. Cuartero, S. et al. Control of inducible gene expression links cohesin to hematopoietic progenitor self-renewal and differentiation. Nat Immunol 19, 932-941 (2018).

16. Schwarzer, W. et al. Two independent modes of chromatin organization revealed by cohesin removal. Nature 551, 51-56 (2017).

17. Rao, S.S.P. et al. Cohesin Loss Eliminates All Loop Domains. Cell 171, 305-320 e324 (2017).

18. Palozola, K.C., Lerner, J. \& Zaret, K.S. A changing paradigm of transcriptional memory propagation through mitosis. Nat Rev Mol Cell Biol 20, 55-64 (2019).

19. Martinez-Balbas, M.A., Dey, A., Rabindran, S.K., Ozato, K. \& Wu, C. Displacement of sequence-specific transcription factors from mitotic chromatin. Cell 83, 29-38 (1995).

20. Xing, H. et al. Mechanism of hsp70i gene bookmarking. Science 307, 421-423 (2005).

21. Kadauke, S. et al. Tissue-specific mitotic bookmarking by hematopoietic transcription factor GATA1. Cell 150, 725-737 (2012).

22. Caravaca, J.M. et al. Bookmarking by specific and nonspecific binding of FoxA1 pioneer factor to mitotic chromosomes. Genes Dev 27, 251-260 (2013).

23. Festuccia, N. et al. Mitotic binding of Esrrb marks key regulatory regions of the pluripotency network. Nat Cell Biol 18, 1139-1148 (2016).

24. Festuccia, N. et al. Transcription factor activity and nucleosome organization in mitosis. Genome Res 29, 250-260 (2019).

25. Ginno, P.A., Burger, L., Seebacher, J., lesmantavicius, V. \& Schubeler, D. Cell cycleresolved chromatin proteomics reveals the extent of mitotic preservation of the genomic regulatory landscape. Nat Commun 9, 4048 (2018).

26. Liu, Y. et al. Widespread Mitotic Bookmarking by Histone Marks and Transcription Factors in Pluripotent Stem Cells. Cell Rep 19, 1283-1293 (2017). 
27. Deluz, C. et al. A role for mitotic bookmarking of SOX2 in pluripotency and differentiation. Genes Dev 30, 2538-2550 (2016).

28. Teves, S.S. et al. A dynamic mode of mitotic bookmarking by transcription factors. Elife 5 (2016).

29. Hsiung, C.C. et al. A hyperactive transcriptional state marks genome reactivation at the mitosis-G1 transition. Genes Dev 30, 1423-1439 (2016).

30. Dey, A., Nishiyama, A., Karpova, T., McNally, J. \& Ozato, K. Brd4 marks select genes on mitotic chromatin and directs postmitotic transcription. Mol Biol Cell 20, 4899-4909 (2009).

31. Blobel, G.A. et al. A reconfigured pattern of MLL occupancy within mitotic chromatin promotes rapid transcriptional reactivation following mitotic exit. Mol Cell 36, 970-983 (2009).

32. Larson, A.G. et al. Liquid droplet formation by HP1alpha suggests a role for phase separation in heterochromatin. Nature 547, 236-240 (2017).

33. Strom, A.R. et al. Phase separation drives heterochromatin domain formation. Nature 547, 241-245 (2017).

34. Gautier, T., Robert-Nicoud, M., Guilly, M.N. \& Hernandez-Verdun, D. Relocation of nucleolar proteins around chromosomes at mitosis. A study by confocal laser scanning microscopy. J Cell Sci 102 ( Pt 4), 729-737 (1992).

35. Ohta, S. et al. Quantitative Proteomics of the Mitotic Chromosome Scaffold Reveals the Association of BAZ1B with Chromosomal Axes. Mol Cell Proteomics 18, 169-181 (2019).

36. Lerner, J. et al. Human mutations affect the epigenetic/bookmarking function of HNF1B. Nucleic Acids Res 44, 8097-8111 (2016).

37. Pallier, C. et al. Association of chromatin proteins high mobility group box (HMGB) 1 and HMGB2 with mitotic chromosomes. Mol Biol Cell 14, 3414-3426 (2003).

38. Dolezel, J., Carter, N. \& Ferguson-Smith, M. Introduction: chromosomes go with the flow. Chromosome Res 12, 1-4 (2004).

39. Ng, B.L. \& Carter, N.P. Laser excitation power and the flow cytometric resolution of complex karyotypes. Cytometry A 77, 585-588 (2010).

40. Gribble, S.M., Ng, B.L., Prigmore, E., Fitzgerald, T. \& Carter, N.P. Array painting: a protocol for the rapid analysis of aberrant chromosomes using DNA microarrays. Nat Protoc 4, 1722-1736 (2009).

41. Brind'Amour, J. \& Lansdorp, P.M. Analysis of repetitive DNA in chromosomes by flow cytometry. Nat Methods 8, 484-486 (2011).

42. Monard, S.P. Chromosome sorting and analysis by FACS. Methods Mol Biol 91, 239254 (1998).

43. Kuipers, M.A. et al. Highly stable loading of $\mathrm{Mcm}$ proteins onto chromatin in living cells requires replication to unload. J Cell Biol 192, 29-41 (2011).

44. van den Boom, V. et al. UTF1 is a chromatin-associated protein involved in ES cell differentiation. J Cell Biol 178, 913-924 (2007).

45. Hakimi, M.A. et al. A chromatin remodelling complex that loads cohesin onto human chromosomes. Nature 418, 994-998 (2002).

46. Tsumura, A. et al. Maintenance of self-renewal ability of mouse embryonic stem cells in the absence of DNA methyltransferases Dnmt1, Dnmt3a and Dnmt3b. Genes Cells 11, 805-814 (2006).

47. Pereira, C.F. et al. ESCs require PRC2 to direct the successful reprogramming of differentiated cells toward pluripotency. Cell Stem Cell 6, 547-556 (2010).

48. Pereira, C.F. et al. Heterokaryon-based reprogramming of human B lymphocytes for pluripotency requires Oct4 but not Sox2. PLoS Genet 4, e1000170 (2008).

49. Guy, J., Hendrich, B., Holmes, M., Martin, J.E. \& Bird, A. A mouse Mecp2-null mutation causes neurological symptoms that mimic Rett syndrome. Nat Genet 27, 322-326 (2001).

50. Della Ragione, F., Vacca, M., Fioriniello, S., Pepe, G. \& D'Esposito, M. MECP2, a multi-talented modulator of chromatin architecture. Brief Funct Genomics 15, 420-431 (2016). 
51. Baubec, T., Ivanek, R., Lienert, F. \& Schubeler, D. Methylation-dependent and independent genomic targeting principles of the MBD protein family. Cell 153, 480-492 (2013).

52. Stadler, M.B. et al. DNA-binding factors shape the mouse methylome at distal regulatory regions. Nature 480, 490-495 (2011).

53. Chen, T. \& Dent, S.Y. Chromatin modifiers and remodellers: regulators of cellular differentiation. Nat Rev Genet 15, 93-106 (2014).

54. Fisher, C.L. \& Fisher, A.G. Chromatin states in pluripotent, differentiated, and reprogrammed cells. Curr Opin Genet Dev 21, 140-146 (2011).

55. Ohta, S. et al. Proteomics Analysis with a Nano Random Forest Approach Reveals Novel Functional Interactions Regulated by SMC Complexes on Mitotic Chromosomes. Mol Cell Proteomics 15, 2802-2818 (2016).

56. Yan, J. et al. Transcription factor binding in human cells occurs in dense clusters formed around cohesin anchor sites. Cell 154, 801-813 (2013).

57. Morales, C. \& Losada, A. Establishing and dissolving cohesion during the vertebrate cell cycle. Curr Opin Cell Biol 52, 51-57 (2018).

58. Waizenegger, I.C., Hauf, S., Meinke, A. \& Peters, J.M. Two distinct pathways remove mammalian cohesin from chromosome arms in prophase and from centromeres in anaphase. Cell 103, 399-410 (2000).

59. Lavagnolli, T. et al. Initiation and maintenance of pluripotency gene expression in the absence of cohesin. Genes Dev 29, 23-38 (2015).

60. Tachibana-Konwalski, K. et al. Rec8-containing cohesin maintains bivalents without turnover during the growing phase of mouse oocytes. Genes Dev 24, 2505-2516 (2010).

61. Naumova, N. et al. Organization of the mitotic chromosome. Science 342, 948-953 (2013).

62. Fussner, E., Ahmed, K., Dehghani, H., Strauss, M. \& Bazett-Jones, D.P. Changes in chromatin fiber density as a marker for pluripotency. Cold Spring Harb Symp Quant Biol 75, 245-249 (2010).

63. Hassan-Zadeh, V., Rugg-Gunn, P. \& Bazett-Jones, D.P. DNA methylation is dispensable for changes in global chromatin architecture but required for chromocentre formation in early stem cell differentiation. Chromosoma 126, 605-614 (2017).

64. Raccaud, M. et al. Mitotic chromosome binding predicts transcription factor properties in interphase. Nat Commun 10, 487 (2019).

65. Buchenau, P., Hodgson, J., Strutt, H. \& Arndt-Jovin, D.J. The distribution of polycombgroup proteins during cell division and development in Drosophila embryos: impact on models for silencing. J Cell Biol 141, 469-481 (1998).

66. Voncken, J.W. et al. Chromatin-association of the Polycomb group protein BMI 1 is cell cycle-regulated and correlates with its phosphorylation status. J Cell Sci 112 ( Pt 24), 4627-4639 (1999).

67. Dietzel, S., Niemann, H., Bruckner, B., Maurange, C. \& Paro, R. The nuclear distribution of Polycomb during Drosophila melanogaster development shown with a GFP fusion protein. Chromosoma 108, 83-94 (1999).

68. Ang, Y.S. et al. Wdr5 mediates self-renewal and reprogramming via the embryonic stem cell core transcriptional network. Cell 145, 183-197 (2011).

69. Di Micco, R. et al. Control of embryonic stem cell identity by BRD4-dependent transcriptional elongation of super-enhancer-associated pluripotency genes. Cell Rep 9, 234-247 (2014).

70. Finley, L.W.S. et al. Pluripotency transcription factors and Tet1/2 maintain Brd4independent stem cell identity. Nat Cell Biol 20, 565-574 (2018).

71. Mishra, B.P., Ansari, K.I. \& Mandal, S.S. Dynamic association of MLL1, H3K4 trimethylation with chromatin and Hox gene expression during the cell cycle. FEBS $J$ 276, 1629-1640 (2009). 
72. Steffen, P.A. et al. Quantitative in vivo analysis of chromatin binding of Polycomb and Trithorax group proteins reveals retention of ASH1 on mitotic chromatin. Nucleic Acids Res 41, 5235-5250 (2013).

73. Schuettengruber, B., Martinez, A.M., lovino, N. \& Cavalli, G. Trithorax group proteins: switching genes on and keeping them active. Nat Rev Mol Cell Biol 12, 799-814 (2011).

74. Gilbert, N. et al. DNA methylation affects nuclear organization, histone modifications, and linker histone binding but not chromatin compaction. J Cell Biol 177, 401-411 (2007).

75. Jimenez-Useche, I. \& Yuan, C. The effect of DNA CpG methylation on the dynamic conformation of a nucleosome. Biophys J 103, 2502-2512 (2012).

76. Jimenez-Useche, I. et al. DNA methylation effects on tetra-nucleosome compaction and aggregation. Biophys J 107, 1629-1636 (2014).

77. Nikitina, T. et al. Multiple modes of interaction between the methylated DNA binding protein MeCP2 and chromatin. Mol Cell Biol 27, 864-877 (2007).

78. Linhoff, M.W., Garg, S.K. \& Mandel, G. A high-resolution imaging approach to investigate chromatin architecture in complex tissues. Cell 163, 246-255 (2015).

79. Kulak, N.A., Pichler, G., Paron, I., Nagaraj, N. \& Mann, M. Minimal, encapsulated proteomic-sample processing applied to copy-number estimation in eukaryotic cells. Nat Methods 11, 319-324 (2014).

80. Cox, J. et al. Accurate proteome-wide label-free quantification by delayed normalization and maximal peptide ratio extraction, termed MaxLFQ. Mol Cell Proteomics 13, 2513-2526 (2014).

81. Tyanova, S. et al. The Perseus computational platform for comprehensive analysis of (prote)omics data. Nat Methods 13, 731-740 (2016).

82. Mastronarde, D.N. Automated electron microscope tomography using robust prediction of specimen movements. J Struct Biol 152, 36-51 (2005).

83. Kremer, J.R., Mastronarde, D.N. \& McIntosh, J.R. Computer visualization of threedimensional image data using IMOD. J Struct Biol 116, 71-76 (1996). 
bioRxiv preprint doi: https://doi.org/10.1101/750067; this version posted August 28,2019 . The copyright holder for this preprint (which was not certified by peer review) is the author/funder. All rights reserved. No reuse allowed without permission.

a
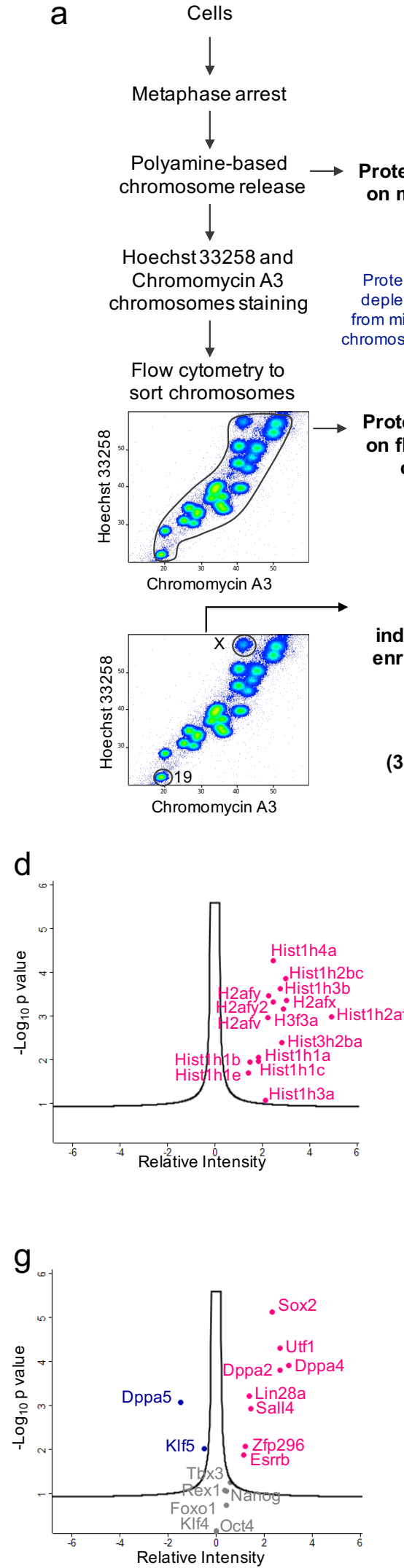

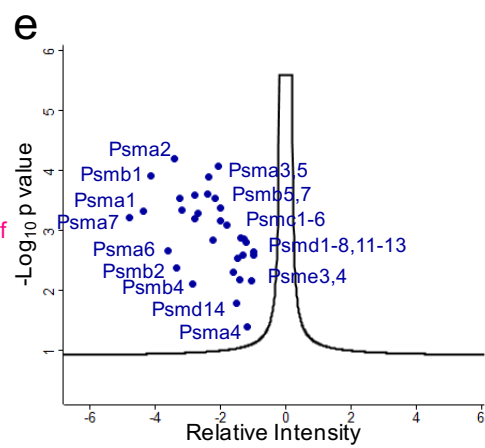

Proteomics LC-MS/MS on flow-sorted mitotic chromosomes

Validate

individual chromosome enrichment and integrity

(3D-SIM and Cryo-ET)

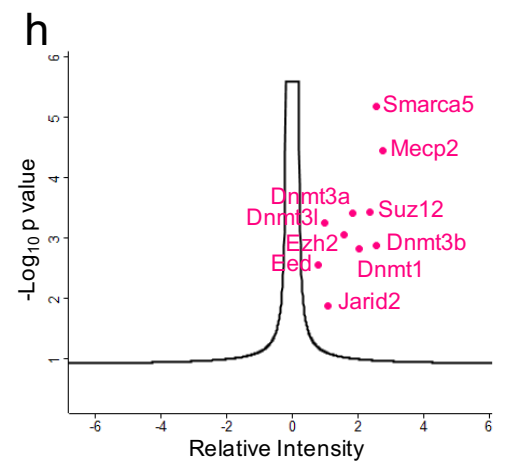

Total mitotic cell lysate proteins $(5888)$

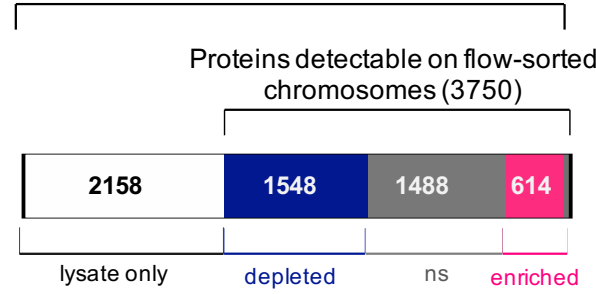

C
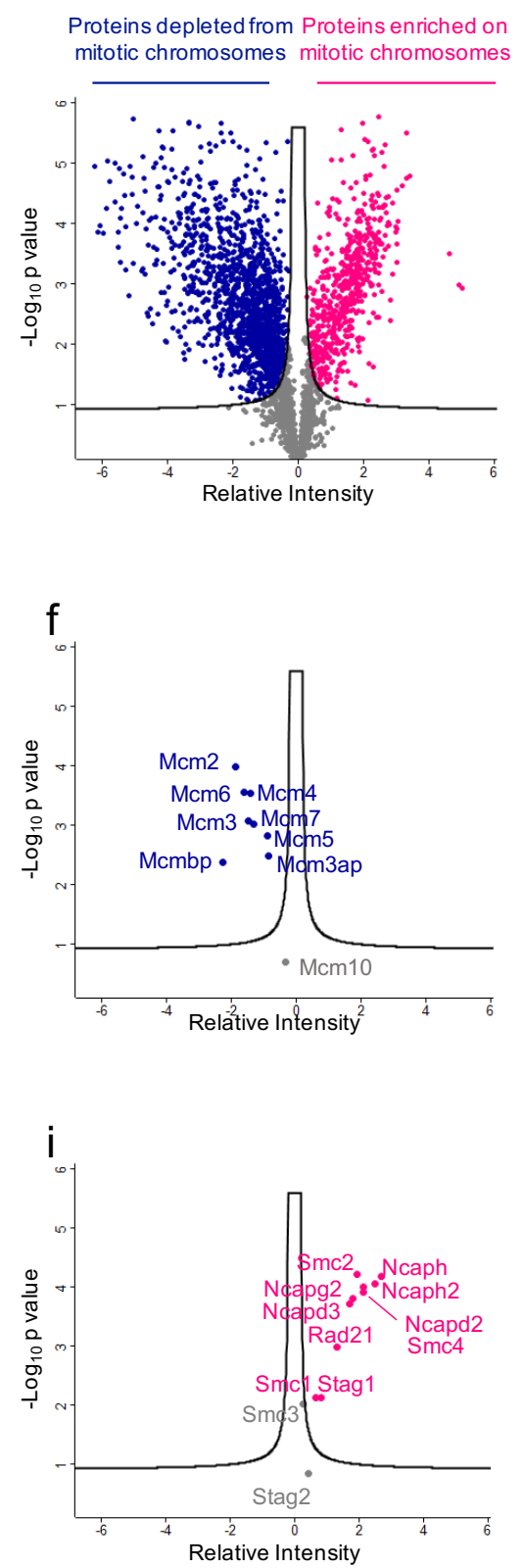

Figure 1. Proteins bound to ESC-derived metaphase chromosomes. (a) Scheme of experimental strategy used to isolate native metaphase chromosomes from ESCs and identify proteins bound to mitotic chromatin. Hoechst33258 and Chromomycin A3 bivariate Karyotype was assessed by flow cytometry and the gates used to sort all chromosomes, chromosomes 19 , or the $\mathrm{X}$ chromosomes are indicated. Proteomic analysis was performed using LC-MS/MS on total mitotic cell lysate, or on flow-purified chromosomes, to identify proteins bound to native metaphase chromosomes. (b) Diagram of number of proteins identified by proteomic analysis as differentially detected in lysates and chromosome sorted samples. (c) Volcano plot of proteins detected as being significantly (FDR<0.01) enriched on sorted chromosomes relative to mitotic lysates. Proteins were plotted by relative intensity (LFQ intensity of Purified chromosomes - LFQ intensity of mitotic lysates) and significance (-Log p) using Perseus software. Volcano plots show (d) histones, (e) components of the proteasome, (f) DNA replication machinery, (g) pluripotency-associated transcription factors, $(\mathrm{h})$ chromatin repressors or (i) SMC associate proteins, that are enriched (red), depleted (blue) or not significantly enriched (grey) on ESC mitotic chromosomes versus mitotic lysates. 

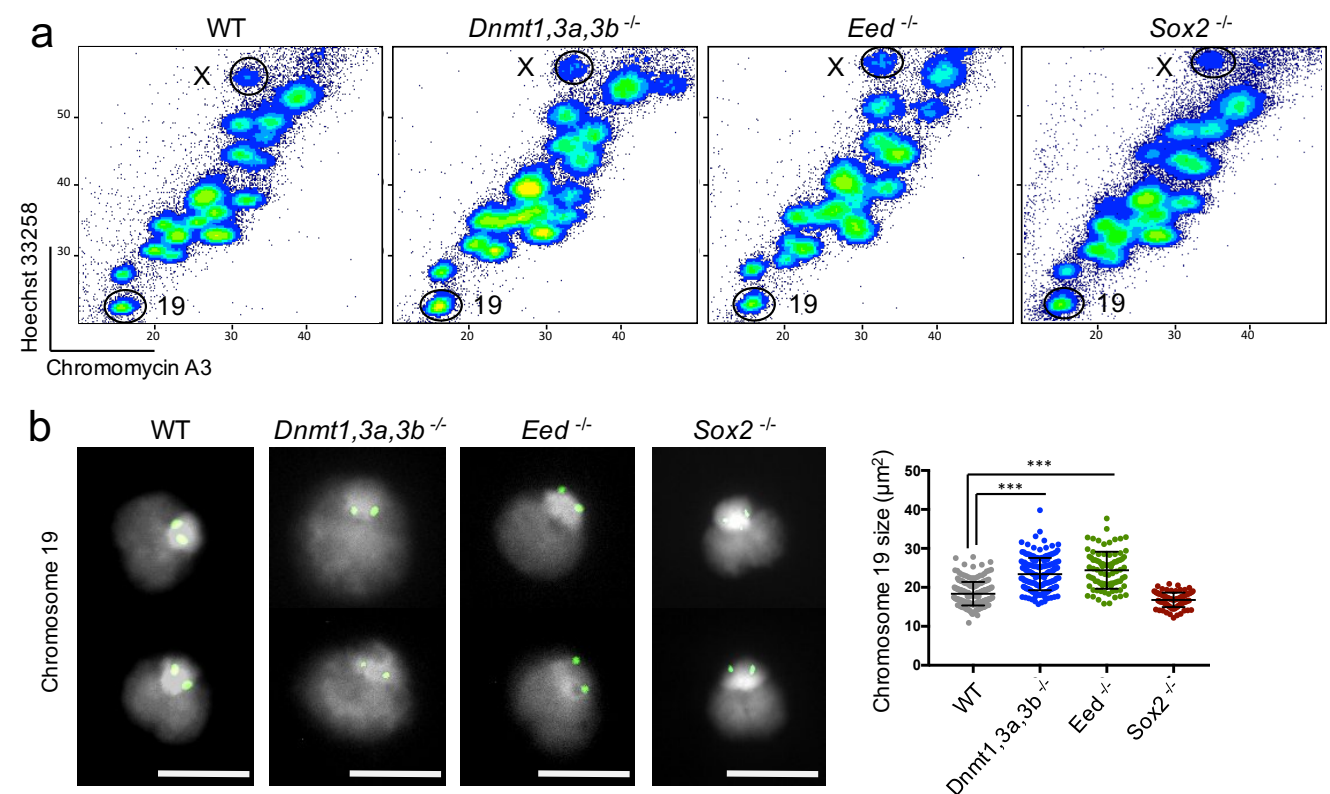

Sox $2^{-1}$


Eed ${ }^{-1}$
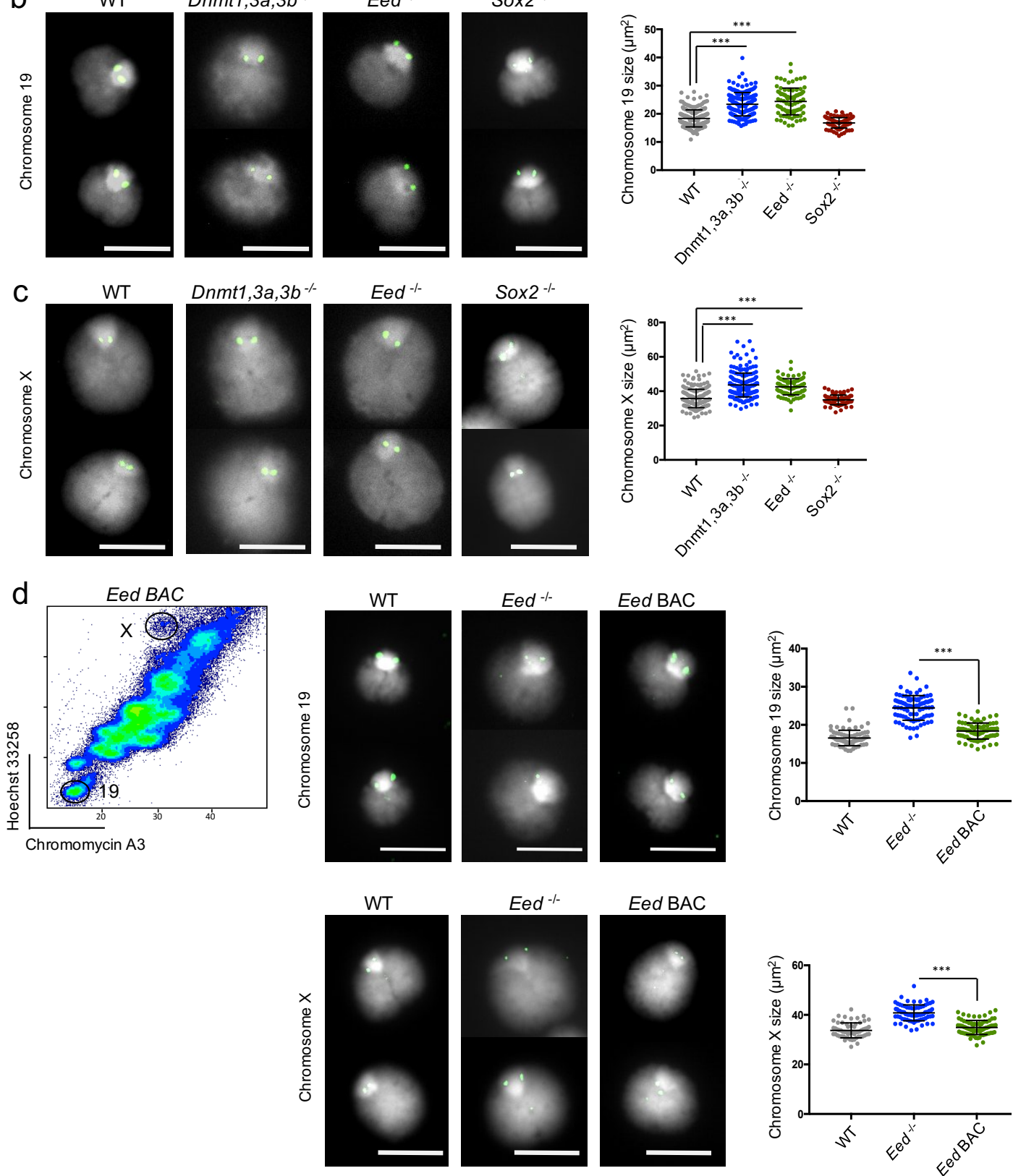

Eed BAC
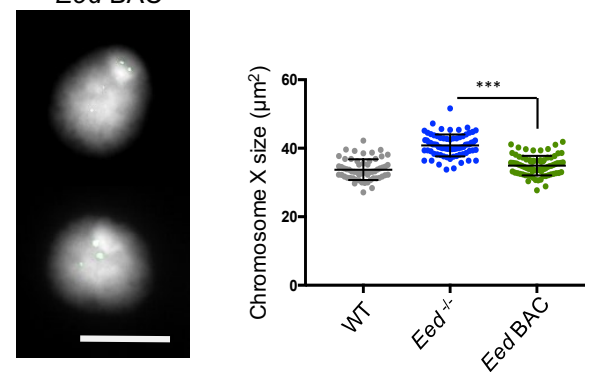

Figure 2. Increased size of ESC mitotic chromosomes that lack DNA methylation or PRC2 activity. (a) Flow karyotype of mitotic chromosomes isolated from WT ESCs or mutant ESCs that lack Dnmt1,3a,3b, Eed or Sox2. Gates used to isolate chromosome 19 or X are indicated. Representative images of mitotic chromosomes 19 (b) and X (c) from different ESCs are shown, where DAPI stain (light grey) and Cenpa label (green) indicate the chromosome body and centromere respectively, scale bar $=5 \mu \mathrm{m}$. Chromosome size was calculated by measuring a minimum of 100 chromosomes for each ESC line, mean \pm SD are indicated. (d) Flow karyotype and mitotic chromosome size of Eed ${ }^{1-}$ ESCs before, and after restoring Eed expression (Eed BAC). Representative images show chromosome 19 (top panel) and $\mathrm{X}$ chromosome (lower panel) isolated from WT, Eed-deficient $\left(\mathrm{Eed}^{-1}\right)$ and rescued (Eed BAC) ESCs, where chromosome size was measured for a minimum of 100 chromosomes for each cell line, and mean \pm SD values are shown. Scale bar=5 $\mu \mathrm{m}$. Asterisks show statistical significance estimated by unpaired two-tailed Student's t test ${ }^{* *}<0.001$. 
a
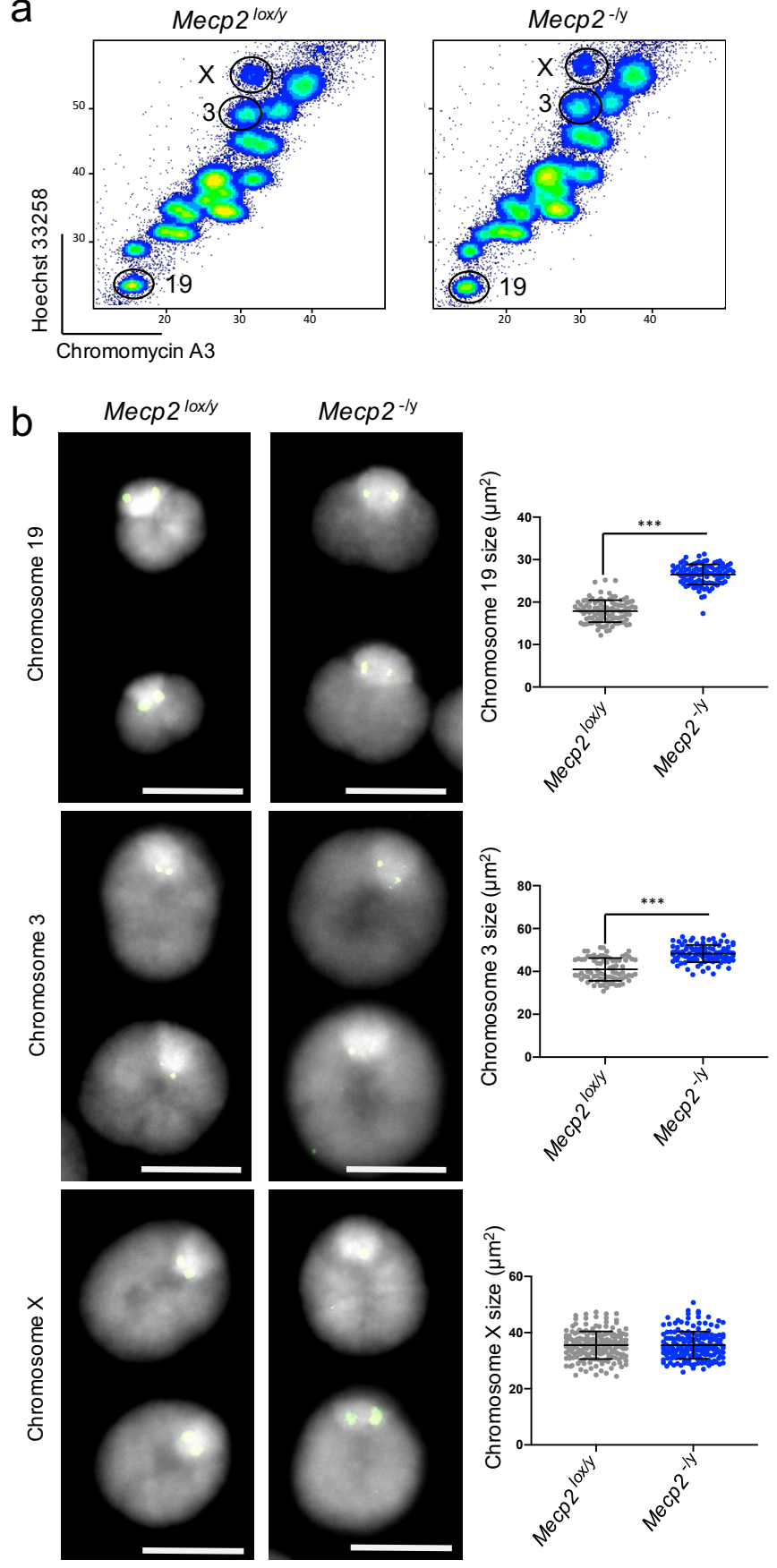

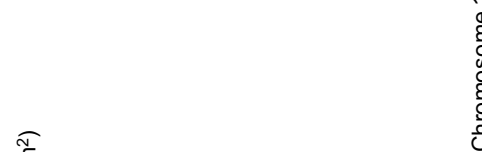

0
0
0
0
0
0
ह
o
ป

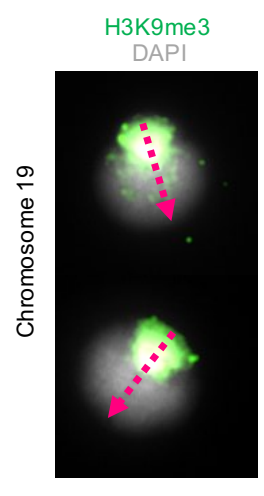

C

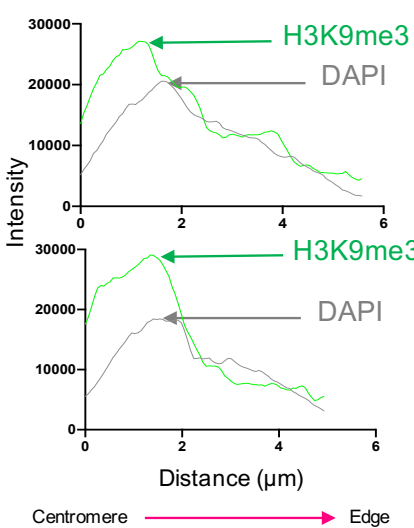

Mecp2-/y
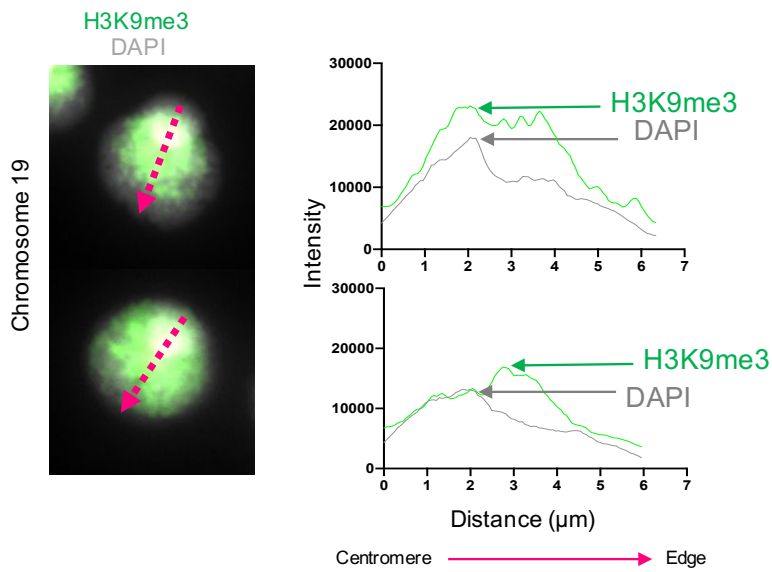

Figure 3. Increased size of mitotic chromosomes in ESCs lacking Mecp2. (a) Flow karyotype of mitotic chromosomes isolated from Mecp2 $2^{l o x / y}$ or Mecp2 $2^{-/ y}$ ESCs. Gates used to isolate chromosome 19, 3 or X are indicated. Representative images of mitotic chromosomes 19, 3, and X (b) from ESCs Mecp2 $2^{\text {lox/y }}$ and Mecp2 ${ }^{-/ y}$ are shown, where DAPI stain (light grey) and Cenpa label (green) indicate the chromosome body and centromere respectively, scale bar $=5 \mu \mathrm{m}$. Chromosome size was calculated by measuring a minimum of 100 chromosomes for each ESC line, mean \pm SD are indicated. Asterisks indicate statistical significance estimated by unpaired two-tailed Student's t test ${ }^{* * *}<0.001$. (c) Representative images of immunofluorescence labelling of histone H3K9me3 (green) and DAPI (light grey) on mitotic chromosome 19 from $M e c p 2^{\text {lox/y }}$ and Mecp2 $2^{-/ y}$ ESCs. Distance distribution plots of H3K9me3 (green) and DAPI (grey) intensities were measured for each chromosome along a central selected axis (red) from the centromere to the distal edge of the chromosome arm. 

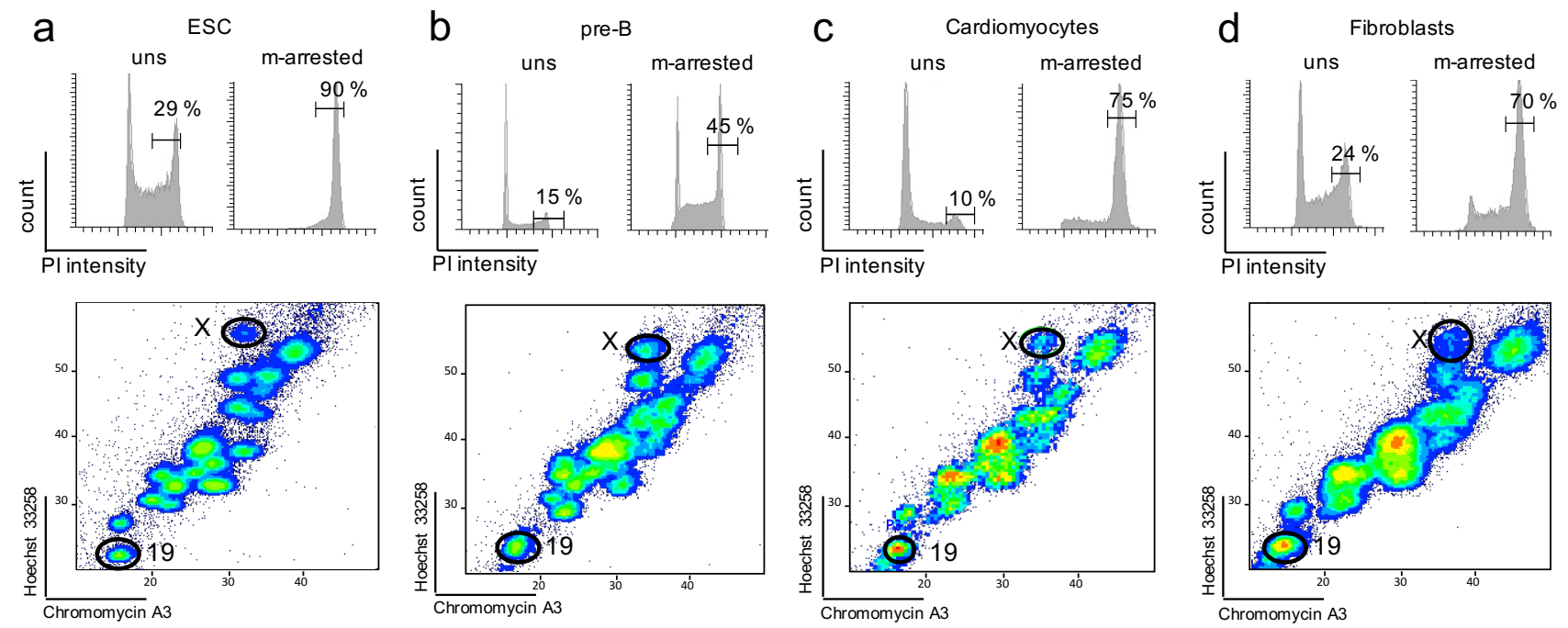

e ESC

pre-B

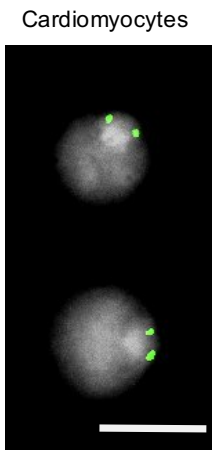

Fibroblasts


Figure 4. Native mitotic ESC chromosomes are larger than equivalents isolated from differentiated cells. Cell cycle profiles of mouse ESCs (a), pre-B cells (b), cardiomyocytes (c) and embryonic fibroblasts (d), were determined by staining with propidium iodide (PI). Left panel shows unsynchronized (uns) cells, right panel shows samples 6-12 hours after treatment with demecolcine (m-arrested), where values indicate percentage cells in G2/M cells. Lower panel shows flow karyotype of demecolcine-treated cells and the gates used to isolate chromosomes 19 and X. Representative images of native mitotic chromosomes 19 (e) and X (f) isolated from mouse ESCs, pre-B cells, cardiomyocytes (HL-1) and embryonic fibroblasts. DAPI stain (light grey) and Cenpa (green) labelling are shown, scale bars $=5 \mu \mathrm{m}$. Chromosome size was determined by measuring chromosome area for a minimum of 100 chromosomes for each cell type, and calculating mean \pm SD. Asterisks indicate statistical significance estimated by unpaired two-tailed Student's test ${ }^{* * *}<0.001$. 
a

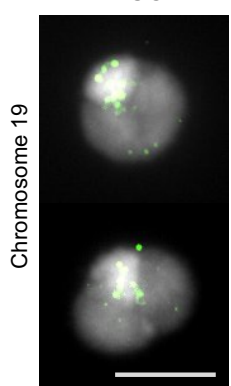

$\operatorname{Rad} 21 / \mathrm{DAP}$
pre-B

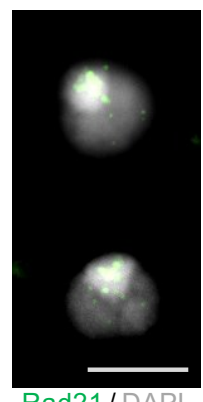

$\operatorname{Rad} 21 / \mathrm{DAPl}$

C

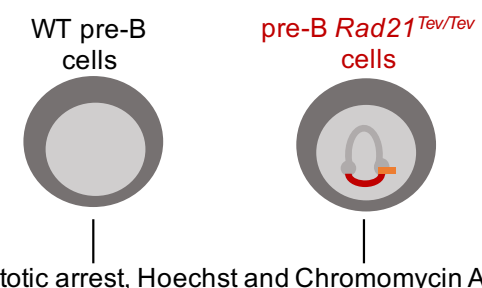

Mitotic arrest, Hoechst and Chromomycin A3 staining, and flow sort of chromosomes 19

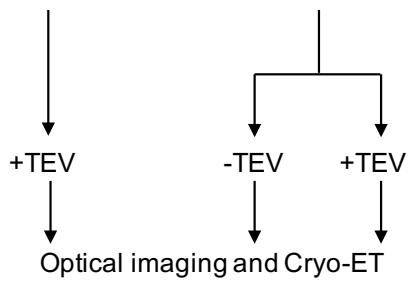

e
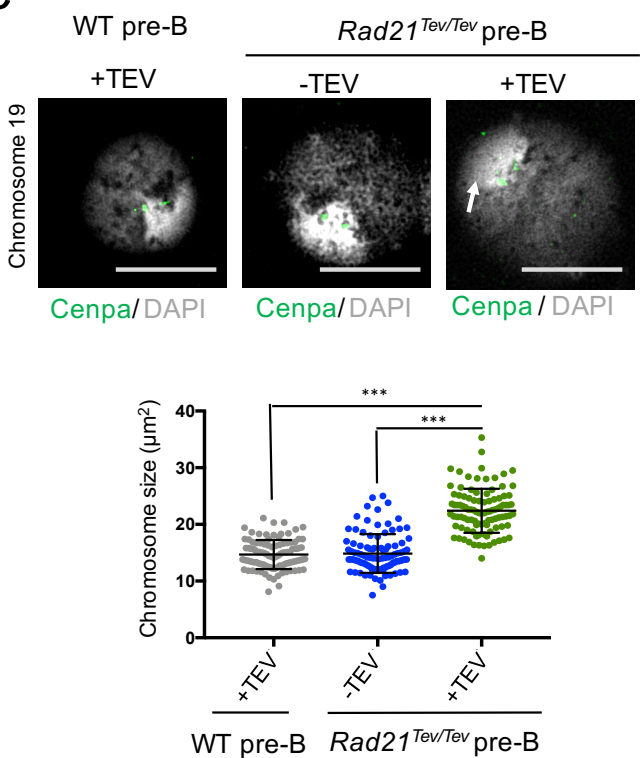

b

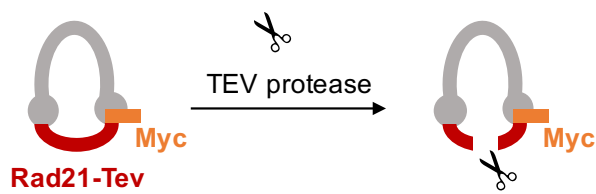

d

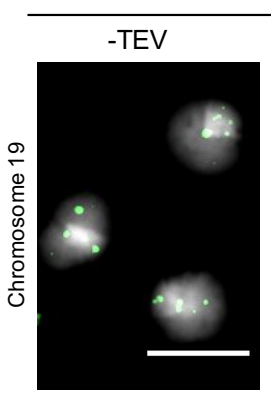

Myc / DAP + TEV

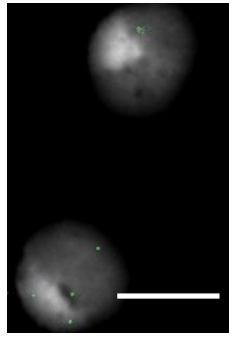

Myc / DAPI
Myc

Mean of fluorescence

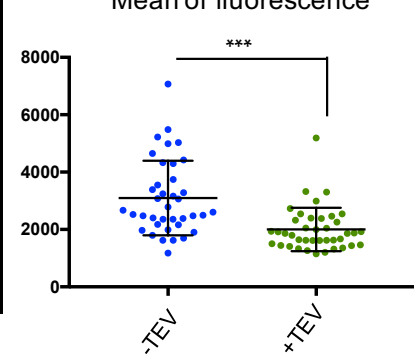

f
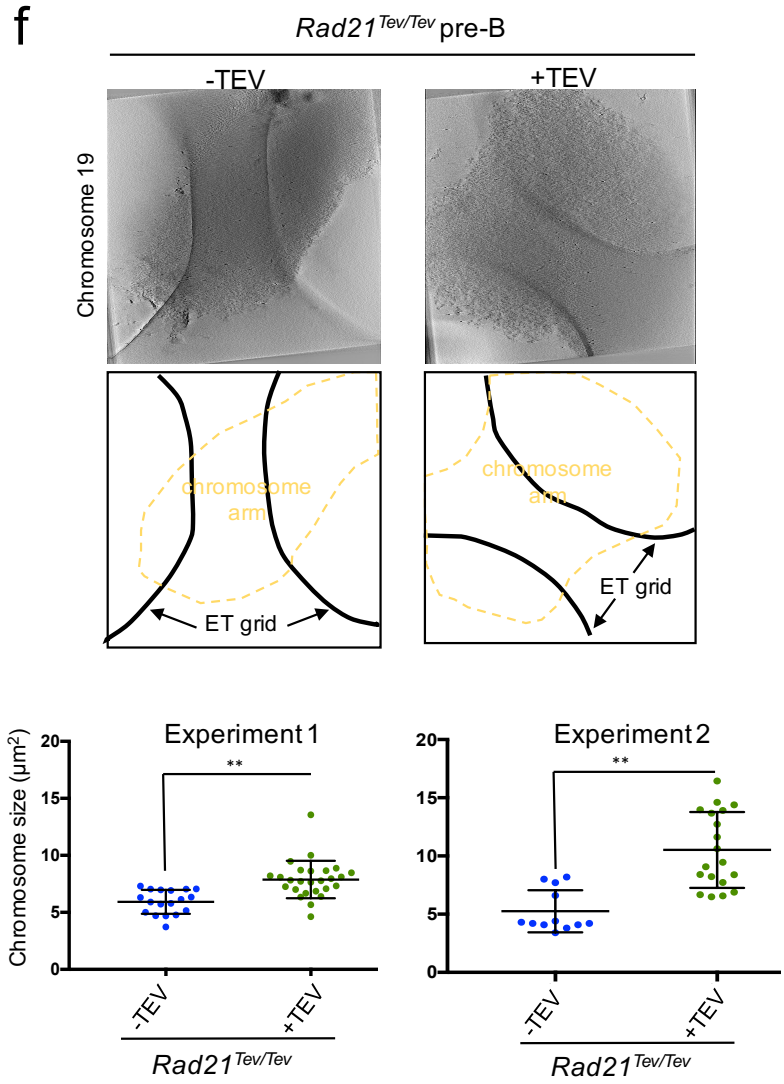

Figure 5. Experimentally induced cleavage of cohesin alters mitotic chromosome size. (a) Rad21 labelling (green) shows cohesin bound to native mitotic chromosome 19 isolated from ESCs or pre-B cells, scale bars $=5 \mu m$. (b) Experimental strategy used to cleave cohesin using TEV protease; illustrated is a cohesin ring containing Smc1, 3, and Rad21-TevMyc. (c) Scheme used to isolate and image mitotic chromosomes from WT and Rad21-Tev-myc pre-B (Rad21 ${ }^{\text {Tev/Tev }}$ ) cells. Mitotic chromosomes of WT pre-B cells or from Rad21 $1^{T e v / T e v}$ pre-B cells were purified by flow cytometry and incubated with or without TEV protease. (d) Myc labeling (green) of Rad21 ${ }^{\text {Tev/Tev }}$ purified chromosome 19 shows reduced Myc levels after treatment with TEV protease (images left, and quantified by intensity, right), scale bars $=5 \mu \mathrm{m}$, where at least 30 chromosomes were examined for each condition and mean intensity values \pm SD are shown. (e) Representative superresolution SIM images of purified mitotic chromosome 19 isolated from WT or Rad21 ${ }^{\text {Tev/TeV }}$ pre-B cells, treated with TEV in situ (+TEV) or with buffer alone (-TEV). Chromosome size was measured for a minimum of 100 chromosomes for each condition, values indicate mean $\pm S D$, scale bars $=2.86 \mu \mathrm{m}$. (f) Representative slices through cryo-electron tomograms (Cryo-ET) of chromosome 19 isolated from Rad $21^{\text {Tev/Tev }}$ pre-B cells and treated with TEV in situ (+TEV) or with buffer alone (-TEV) (top panel) and Cryo-ET image explanation (middle panel). Graphs show chromosome size, calculated as area measurements from 2D electron microscopy images. Values from two independent experiments are shown. Asterisks indicate statistical significance estimated by unpaired two-tailed Student's $t$ test. ${ }^{* * *}<0.001,{ }^{* *}<0.01$. 